\title{
Rehabilitation after COVID-19: an evidence-based approach
}

\author{
Author: Derick T Wade ${ }^{A}$
}

\begin{abstract}
After severe COVID-19 disease, many patients will experience a variety of problems with normal functioning and will require rehabilitation services to overcome these problems. The principles of and evidence on rehabilitation will allow an effective response. These include a simple screening process; use of a multidisciplinary expert team; four evidence-based classes of intervention (exercise, practice, psychosocial support, and education particularly about self-management); and a range of tailored interventions for other problems. The large number of COVID-19 patients needing rehabilitation coupled with the backlog remaining from the crisis will challenge existing services. The principles underpinning vital service reconfigurations needed are discussed.
\end{abstract}

KEYWORDS: COVID-19, rehabilitation, evidence, service reconfiguration

DOI: $10.7861 /$ clinmed.2020-0353

\section{Introduction}

How should patients needing rehabilitation after COVID-19 be identified? What rehabilitation interventions will benefit the patient? How will rehabilitation services need to change?

Several policy documents on the subject of rehabilitation following COVID-19 have been published, but only very limited and incomplete evidence is available to support the conclusions drawn because there has been no time for evaluative research. ${ }^{1-3}$ An early systematic review of residual problems is equally incomplete, due to lack of evidence. ${ }^{4}$

Medical services have responded effectively to the COVID-19 crisis, despite a similar lack of direct evidence. This article shows that rehabilitation can also provide evidence-based interventions based on existing evidence. It does not focus on specific professions or treatments, as each patient's needs are different, but does explain what should be done, and why.

The article is aimed at doctors not involved in rehabilitation, who may have relatively limited understanding of rehabilitation - the process, the interventions, and the benefits that may arise. It is

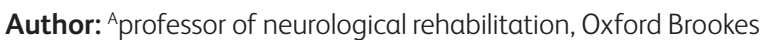
University, Oxford, UK also relevant to other healthcare professionals and to patients and their families. Its goals are to:

$>$ set the context, discussing how the medical aspects of COVID-19 relate to rehabilitation, and when referral to rehabilitation is appropriate

> discuss the interventions rehabilitation services could give, and the supporting evidence

> consider how COVID-19 might precipitate change and improve UK rehabilitation services so that most COVID-19 patients could receive rehabilitation.

Two further assumptions underlie the article:

> It considers only patients who have had COVID-19 of sufficient severity to seek medical help and who have continuing problems or concerns related to COVID-19.

> Rehabilitation refers to a multidisciplinary team having expert knowledge and skills; it is not synonymous with so-called 'specialist rehabilitation'. ${ }^{5}$

\section{The need for rehabilitation following COVID-19}

COVID-19's impact arose from its rapid emergence, the number of people needing intensive care, and the lack of prior knowledge of its manifestations. COVID-19 patients presented many clinical problems, including respiratory failure, excessive immunological response and clotting disorders, renal failure and myocarditis. Medical services responded by drawing on evidence, not specifically derived from patients with COVID-19 but directly applicable to their problems. Over time, some techniques, such as lying patients prone to assist breathing and using continuous positive airway pressure, have been found to be more effective than expected. Existing knowledge has been used successfully. Knowledge of the disease process and which tissues are likely to have been damaged is important in the rehabilitation process. The disease determines both what impairments are likely or unlikely, guiding assessment, the general prognosis and planning of rehabilitation. The whole range of individual problems arising after COVID-19 and their relative frequency is not yet known. Nevertheless, apart from its effects upon the respiratory system, the virus can affect the heart and cardiovascular system, ${ }^{6}$ the brain directly (encephalitis) and indirectly (eg secondary to hypoxia or vascular thrombosis), ${ }^{7}$ the kidney and renal function, ${ }^{8}$ blood clotting ${ }^{9}$ and the gastrointestinal tract:; ${ }^{10}$ the virus has also been found in semen. ${ }^{11}$ 
Table 1. Areas that should be considered when reviewing the situation of a person with COVID-19

\section{Question}

\section{Initial question:}

Compared with before you had COVID-19, are there any new problems or symptoms concerning you?

\section{Activity domains:}

Are you able to undertake, if you wish and at the level you expect:

> Vocational activities (work, education, other occupation)?

$>$ Leisure activities (active and/or sedentary)?

$>$ Shopping and other community activities, including driving?

$>$ Household and domestic activities?

$>$ Feeding yourself, swallowing safely?

> Washing, dressing, using toilet, being continent?

$>$ Getting about the house (including stairs), moving in and out of the house, and getting around outdoors on foot or using transport?

> Social interaction with others - talking, taking part in conversations etc?

> Maintaining your relationships, eg with partner, family or friends?

\section{Common symptoms:}

Do you have (new) problems with:

> Fatigue, endurance, being overtired?

> Sense of smell and taste?

> Pain or painful abnormal sensations?

$>$ Control over your bladder or bowels?

$>$ Vision and your eyes?

$>$ Ears and hearing?

$>$ Thinking, remembering?

$>$ Your mood?

\section{Check}

Are there any other problems in your daily life that concern you?

\section{Comment}

A neutral first question, sufficient in many instances. Wait until all problems and symptoms have been revealed

Only ask about those appropriate to the person

If someone is (back) at work full-time, other problems are unlikely Gardening, reading, socialising

Ask about remembering and organising shopping or travel

Cooking, housework, repairs

Check on taste and enjoyment

Bath or shower

Ask about falls if appropriate

Includes hearing

Be alert to changes in temper, and changes not expected

More than before?

Advise a smoke alarm if no smell

May be appropriate occasionally
We therefore have to assume that, after COVID-19, a patient may develop persisting dysfunction of almost any organ system and thus have almost any symptoms and signs. This is similar to the situation with many other disabling conditions, including trauma, systemic lupus erythematosus, diabetes and meningococcal septicaemia. Patients with COVID-19 are more likely to have pre-existing disabling conditions and, in addition, will experience the well-established direct (physical) and indirect (psychological) effects associated with severe illness and a long stay in an intensive care unit. ${ }^{12}$

There is no specific symptom, or group of symptoms or signs, that indicates the overall severity of the COVID-19 illness. This is in contrast to stroke, for example, where markers such as the National Institute of Health Stroke Scale (NIHSS) score measures, to an extent, the severity of stroke, its prognosis and outcome. The situation with COVID-19 is more like that after trauma, where there is very little relationship between the index of trauma severity the injury severity score (ISS) - and the need for rehabilitation.

The complexity and variability of the damage caused by COVID-19, coupled with the pre-existing disabling long-term conditions that many patients will have, means that there is no single, COVID-19 specific method to determine the need for rehabilitation. This is actually the situation for almost all diseases, and a generic method for identifying people who might benefit from rehabilitation should be used. Because almost any person with an ongoing disability who has not been seen within a rehabilitation service is likely to benefit, ${ }^{13}$ the method can be reduced to discovering whether the patient has any ongoing problems or concerns. This includes pre-existing problems.

There is no validated generic checklist for any condition, but Table 1 shows a reasonable checklist. If the person does not admit to any problems, ideally asked in the presence of a family member, then it is unlikely that a more detailed rehabilitation assessment is needed. If a problem or concern is identified and is not obviously irresolvable or going to resolve without intervention soon, then the patient should be referred to a full, multidisciplinary rehabilitation service. The patient should not simply be referred to a uniprofessional service (eg physiotherapy), but referral to a single profession within a multidisciplinary service is satisfactory.

\section{Applying the principles of rehabilitation to COVID-19}

A definition of rehabilitation is given in Box 1. This is derived from a review of evidence, ${ }^{13}$ and is set within Donabedian's structure- 
process-outcome framework. ${ }^{14}$ Rehabilitation is explained in more detail elsewhere. ${ }^{15-20}$

Just as with acute medical care, rehabilitation has no evidence specific to COVID-19 but, just as with medical care, individual problems likely to be seen after COVID-19 are all quite common and well-studied. Rehabilitation can draw on this large evidence base and, using existing skills, can (given adequate resources) offer effective rehabilitation for almost all patients needing it. Just as with acute medical management, some specific problems needing specific treatments may in time be identified, though it is unlikely that they will be unique to COVID-19.

Rehabilitation is, like medical care, a problem-solving process, ${ }^{16,17}$ with much evidence supporting its effectiveness. ${ }^{13,15}$ It starts with a diagnostic process (assessment) aiming to discover what the patient's primary problems and concerns are, and to understand how they arise and how they may be ameliorated. This assessment is undertaken using the holistic biopsychosocial model of illness as a framework ${ }^{19}$ and will usually require a multidisciplinary team with suitable expertise. ${ }^{20}$

It is likely that, after COVID-19, the majority of, but not all, patients will need pulmonary rehabilitation. Pulmonary rehabilitation in the UK is usually a separate service attached to a respiratory medicine department. It will rarely have the breadth of knowledge and skills required to identify and diagnose the many other potential problems arising after COVID-19 (eg critical illness neuromyopathy), let alone problems related to preexisting conditions and disabilities. Other rehabilitation services will be needed if all problems are to be identified and treated successfully.

Rehabilitation planning follows assessment and formulation. Planning is based on the patient's wishes and values, coupled with knowledge about prognosis and available interventions, and sets long-term goals with intermediate and short-term goals. ${ }^{21}$ At present, detailed knowledge of prognosis after COVID-19 is missing. Until shown otherwise, decisions should be made on the premise that long-term irreversible tissue damage will be relatively rare, and that recovery of most problems will occur over 12-24 months. This is the pattern after most acute onset disorders.

Effective rehabilitation interventions fall into five categories that are the same across all conditions (see Box 1).13 Therefore, effective rehabilitation interventions for patients after COVID-19 can be given on a reasonably sound evidential basis.

Exercise that uses muscles and increases demand upon the cardio-respiratory system will be important. It benefits not simply general fitness, but also a range of other problems such as fatigue, emotional disturbance, lack of confidence, and performance of effortful activities such as walking, if these were limited. It is a core component of all cardiac and pulmonary rehabilitation and should be encouraged from the outset.

Specific practice at activities that are limited in some way is the key step to improvement in those activities. In rehabilitation, as in all other aspects of life, the maxim 'practice makes perfect' holds true, even if perfection is not achieved. In rehabilitation jargon, it is referred to as 'task-specific training'. The principle applies in all conditions. It will be particularly relevant to COVID-19 patients with neurological and musculoskeletal damage.

Psycho-social support is the third intervention identified in almost all studies showing rehabilitation to be effective. It is not well-defined, but refers most commonly to management of emotional disturbance, changes in self-esteem and self-
Box 1. An evidence-based description of effective rehabilitation ${ }^{13}$ based on the framework of Donabedian $^{14}$

\section{Patients and places}

Rehabilitation may benefit anyone with a longer-term disabling illness at any stage of that illness and be delivered in any setting.

\section{The structure}

The necessary resource is a multidisciplinary team with the range of knowledge and skills needed to manage, from within their own team, $80 \%$ of patients seen without additional help. This team should be based within a single organisation and have its own geographic base, and financial arrangements should be managed in consultation with the team. ${ }^{15}$

\section{The goal (outcome)}

To optimise a patient's self-rated quality of life and degree of social integration through optimising independence in activities, minimising pain and distress, and optimising the ability to adapt and respond to changes in circumstances.

\section{The process}

Rehabilitation is a problem-solving process, framed in the context of the holistic biopsychosocial model of illness, delivered in a person-centred way, ${ }^{11}$ and requiring:

$>$ an expert, multidisciplinary team, setting collaborative teambased goals

$>$ a formulation of the situation, covering all domains of the biopsychosocial model

> close, collaborative working across all boundaries, professional, organisational and geographic

> ongoing monitoring of change and effects of interventions

Interventions fall into five categories:

$>$ General exercise that increases cardio-respiratory work

$>$ Repeated practice of functional activities

$>$ Psycho-social therapies

$>$ Education with an emphasis upon self-management

$>$ A set of specific actions tailored to the patient's priorities, needs and goals, covering (if necessary) all domains of the biopsychosocial model of illness, and being evaluated regularly for their benefits and harms, to determine whether they should be continued, changed or abandoned.

confidence, and similar constructs. It involves techniques such as cognitive behavioural therapy and motivational interviewing. Re-establishing social contacts and social networks is also included within this portmanteau word, often involving the arrangement or provision of social support through day centres, social prescribing etc.

The fourth major pillar of all rehabilitation is education. This covers many specific areas: patient self-management; carers (family and professional) being taught how to support self-management; carers being taught to facilitate practice, and/or to provide care safely; carers being encouraged to facilitate social integration; teaching patients, and others as appropriate, about the disease and its management; and setting expectations for all parties. 
For patients after COVID-19, advising on the prognosis and setting expectations will be difficult, and clinicians will have to admit a degree of uncertainty. Therefore, teaching patients and families how to manage this uncertainty will be a particularly important aspect of rehabilitation after COVID-19.

The last characteristic of effective rehabilitation is the personalisation of the process to the individual patient's needs, wishes, values and circumstances. It starts with making the interventions in the preceding four domains relevant to the individual patient. A standard approach cannot be suitable for all patients.

More importantly, personalisation also refers to identifying and using other specific interventions outside the four pillars. Many are obvious, and concern assistive technology and adaptation of the environment, such as providing a wheelchair for one patient who cannot walk, and a prosthetic leg to another. Palliative symptom control, which is integral to rehabilitation, is another important example.

An expert rehabilitation service should also be inventive and alert to possible specific solutions to specific problems. For example, a person who has difficulty reading due to a right visual field loss (after stroke) can read by turning the book upside down, reading into the intact visual field.

The last stage in the reiterative rehabilitation process is evaluation. Have goals been met? If so, are there any further or new goals? If goals have not been achieved, why not and can some alternative intervention be tried? Is it appropriate to transfer care to a less specialised and/or more local service? Does the patient still need to see anyone, or can they be discharged?

Although evidence is available for all these interventions, there is also obviously much to learn about rehabilitation after COVID-19, including the following:

> Are some existing interventions particularly effective, or harmful?

$>$ Which symptoms are particularly common, and what is the optimal treatment strategy?

> What are realistic expectations to give a patient?

$>$ Are there specific late or rare complications to be considered?

\section{Reconfiguring rehabilitation services after COVID-19}

COVID-19 has challenged acute hospital services. They have rapidly adapted, as doctors learned about the disease and its management, and traditional, accepted practice has changed in many areas of medicine. ${ }^{22,23}$ Patients with other health conditions were given lower priority and were disadvantaged, but so far there has been more-or-less sufficient capacity.

COVID-19 also challenged rehabilitation services. Many services were reduced, to release healthcare staff for acute services, and to minimise the risk to patients, many of whom are at higher risk. Rehabilitation has also adapted, for example developing both remote assessment and remote treatment services.

COVID-19 is predicted to challenge rehabilitation a second time, ${ }^{4}$ with many more patients needing longer-term complex rehabilitation input. Unfortunately, even before this crisis, most patients needing rehabilitation did not receive it. ${ }^{15,24}$ Unless there is dramatic change, many people with residual problems after COVID-19 are unlikely to receive expert rehabilitation assessment, advice, and treatments. One obvious reason is the lack of resources, especially but not only trained rehabilitation doctors and clinical psychologists. This cannot be reversed quickly. A less obvious reason is the lack of any sustained interest in the provision and commissioning of rehabilitation. The organisation and commissioning of rehabilitation services is chaotic, without any coherent strategy. For example, partial rehabilitation is provided by very many commissioned services, including specialist rehabilitation; ${ }^{2}$ intermediate care; early supported discharge; discharge to assess; enablement; reablement; home care; single point of access; and young disabled services. In addition, named disease services are commissioned for conditions including stroke, chronic pain (such as back/spinal pain), chronic fatigue, functional neurological disorders, cardiac disease and pulmonary disease. There are many commissioned specialist nurses or therapists attempting to coordinate care between these many services.

Even the terminology is confusing. Rehabilitation is a specialised service, just as a medical specialty like cardiology is. It has specific expertise. 'Specialised rehabilitation' is commissioned solely for resource-intense patients. ${ }^{5}$ It is no more or less expert than other rehabilitation.

Each service is separate, usually time-limited, and usually working to strict criteria. Most services are small, lack senior experienced staff in all professions needed, and often are only funded for a short while, until the next reorganisation or initiative. Many patients are unable to access any service, as there is no comprehensive or coherent framework to ensure full coverage. Some patients move from one service to another as they enter or leave the artificial boundaries imposed.

COVID-19 has precipitated changes in healthcare at an unprecedented scale and speed, both in terms of clinical practice (eg telemedicine) ${ }^{22}$ and in service design, delivery, funding and collaboration. ${ }^{23}$ It is vital that rehabilitation is included in the major changes that will inevitably occur throughout healthcare.

As a specific example, COVID-19 patients will provide a great challenge to the existing structures of rehabilitation. Many COVID-19 patients will need local, community-based rehabilitation services which are weak and often transient. ${ }^{25}$ Most attention is paid to nationally funded highly specialised Level I and IIa inpatient services, ${ }^{5}$ which see only a very small proportion (estimated $1 \%$ ) of all patients needing rehabilitation. For example, patients who have multiple sclerosis and quite severe complex disability requiring specialised services often cannot access rehabilitation. ${ }^{24}$ National, specialised commissioning does not consider such patients, ${ }^{5}$ and local clinical commissioning groups often do not support the expert rehabilitation service needed.

Large numbers of people are surviving COVID-19 after quite severe illness. Although the nature, extent, and frequency of problems is not known, the numbers will hugely exceed the capacity of current commissioned rehabilitation in the UK. More importantly, many patients will present with a mixture of cardio-respiratory, mental health, and neuromuscular problems. Currently this would mean attending at least two separately commissioned services.

One solution that may be suggested, but must be avoided would be to set up a 'COVID-19 rehabilitation service'. This would simply exacerbate the fragmentation of services. Further, trauma patients, patients with multiple sclerosis and many other patient groups would increase their demands for unique services. 
The reasons change is needed, and many of the changes required, have been set out elsewhere. ${ }^{18}$ The main changes needed are:

$>$ rationalisation and reorganisation of the myriad of services for people with continuing disability into a coherent, comprehensive rehabilitation service

$>$ ensuring that every patient with persistent disability is seen by the rehabilitation service from the outset, preferably from first contact with healthcare

$>$ providing the service in all settings from intensive care through hospitals and care homes into the wider community, in parallel with medical services

$>$ providing rehabilitation across all ages and conditions

$>$ ensuring full integration between mental health services and rehabilitation services

$>$ a parallel reconstruction of commissioning, reducing the emphasis on 'specialist rehabilitation' by recognising that all rehabilitation requires expert knowledge and skills.

These changes will require some time, and some resource, but would transform rehabilitation far more quickly that waiting for more resources, because existing resources would be used much more effectively.

This greatly enlarged rehabilitation service will need internal organisation, but this will adapt to local circumstances and need. It will need to be fully integrated into existing disease-based services. It will encompass almost all allied health professionals (including a significant number of nurses).

Every person should work as part of a full multidisciplinary team with rehabilitation expertise, recognising that individual members may work much of the time within other medical teams. Teams and services will need to be organised around both existing medical organisation of services and areas of knowledge and skill that make sense within rehabilitation, not simply around classification of disease, disability or intervention.

It is probable that the number of patients with COVID-19 will be enough to warrant, for a while, a team within the rehabilitation service who would develop specific expertise in the rehabilitation of people who have had COVID-19. This would be similar to having a team with expertise in spinal cord injury, or specialist nurses/ therapists particularly involved with people with Huntington's disease, a model that works well in some areas.

\section{Conclusion}

COVID-19 has challenged all healthcare, including rehabilitation, and will continue to do so for at least a few years. COVID-19 has precipitated rapid change and adaptation. It could precipitate a long-needed change in the attention paid to rehabilitation, leading to a much better organisation and system of funding to allow more efficient and effective use of resources currently working in a fragmented, inefficient way. This will benefit all patients and all parts of healthcare. A phoenix may yet arise from the ashes. ${ }^{1}$

\section{Supplementary material}

Additional supplementary material may be found in the online version of this article at www.rcpjournals.org/clinmedicine:

S1 - Mind map: Rehabilitation and COVID-19.

\section{References}

1 British Society of Rehabilitation Medicine. Rehabilitation in the wake of Covid-19. A phoenix from the ashes. BSRM, 2020. www.bsrm.org.uk/ downloads/covid-19bsrmissue1-published-27-4-2020.pdf [Accessed 27 April 2020].

2 Alberta Health Services. COVID-19 Scientific Advisory Group Rapid Evidence Report. AHS, 2020. www.albertahealthservices.ca/assets/ info/ppih/if-ppih-covid-19-sag-rehabilitation-needs-rapid-review.pdf [Accessed 19 May 2020].

3 Chartered Society of Physiotherapy. Rehabilitation and Covid-19CSP policy statement. CSP, 2020. www.csp.org.uk/professional-dinical/ improvement-innovation/community-rehabilitation/rehab-covid-19policy-statement [Accessed 4 May 2020].

4 Ahmed H, Patel K, Greenwood D et al. Long-term clinical outcomes in survivors of coronavirus outbreaks after hospitalisation or ICU admission: a systematic review and meta-analysis of follow-up studies. medrxiv 2020, 2020.04.16.20067975.

5 NHS England. NHS standard contract for specialist rehabilitation for patients with highly complex needs (all ages). NHSE, 2013. www.england.nhs.uk/wp-content/uploads/2014/04/d02-rehabpat-high-needs-0414.pdf.

6 Siripanthong B, Nazarian S, Muser D et al. Recognizing COVID-19related myocarditis: the possible pathophysiology and proposed guideline for diagnosis and management. Heart Rhythm 2020, in press (doi: 10.1016/j.hrthm.2020.05.001).

7 Helms J, Kremer S, Merdji H et al. Neurologic features in severe SARS-CoV-2 infection. N Engl J Med, in press (doi: 10.1056/ NEJMc2008597).

8 Farkash EA, Wilson AM, Jentzen JM. Ultrastructural evidence for direct renal infection with SARS-CoV-2. J Am Soc Nephrol, in press (doi: 10.1681/ASN.2020040432)

9 Barrett CD, Moore HB, Yaffe MB, Moore EE. ISTH interim guidance on recognition and management of coagulopathy in COVID-19: A comment. J Thromb Haemost 2020, in press (doi: 10.1111/jth.14860).

10 Smyk W, Janik MK, Portincasa P et al. COVID-19: focus on the lungs but do not forget the gastrointestinal tract. Eur J Clin Invest 2020;e13276.

11 Li D, Jin M, Bao P, Zhao W, Zhang S. Clinical characteristics and results of semen tests among men with coronavirus disease 2019. JAMA Netw Open 2020;3:e208292.

12 Jensen JF, Thomsen T, Overgaard D et al. Impact of follow-up consultations for ICU survivors on post-ICU syndrome: a systematic review and meta-analysis. Intensive Care Med 2015;5:763-75.

13 Wade DT. What is rehabilitation? An empirical investigation leading to an evidence-based description. Clin Rehabil 2020;34:571-83.

14 Donabedian A. The evaluation of medical care programs. Bull N Y Acad Med 1968:44:117-24.

15 Wade DT. Rehabilitation - a new approach: Overview and part one: the problems. Clin Rehabil 2015;29:1041-50.

16 Wade DT. Rehabilitation - a new approach. Part two: the underlying theories. Clin Rehabil 2015;29:1145-54.

17 Wade DT. Rehabilitation - a new approach. Part three: the implications of the theories. Clin Rehabil 2016;30:3-10.

18 Wade DT. Rehabilitation - a new approach. Part four: A new paradigm, and its implications. Clin Rehabil 2016;30:109-118.

19 Wade DT, Halligan PW. The biopsychosocial model of illness: a model whose time has come. Clin Rehabil 2017;31:995-1004.

20 Wade DT. A teamwork approach to neurological rehabilitation. In: Dietz V, Ward NS (eds), Oxford Textbook on Neurorehabilitation, $2^{\text {nd }}$ edn. Oxford University Press, 2020:9-22.

21 Siegert RJ, Levack WMM (eds). Rehabilitation goal setting: theory, practice and evidence. CRC Press, 2015.

22 Hollander JE, Sites FD. The transition from reimagining to recreating health care is now. N Engl J Med Catalyst 2020, in press (doi: 10.1056/CAT.20.0093). 
23 Berwick DM. Choices for the "New Normal". JAMA 2020, in press (doi: 10.1001/jama.2020.6949)

24 Royal College of Physicians. National Audit of Services for People with Multiple Sclerosis 2008. RCP, 2008. www.rcplondon.ac.uk/ projects/outputs/national-audit-services-people-multiple-sclerosisarchived-2008-reports.

25 Enderby P, Wade DT. Community rehabilitation in the United Kingdom. Clin Rehabil 2001;15:577-81.
Address for correspondence: Prof Derick T Wade, OxINMAHR and Movement Science Group, Faculty of Health and Life Sciences, Oxford Brookes University, Headington Campus, Gypsy Lane, Oxford OX3 OBP, UK.

Email: derick.wade@ntlworld.com

\section{[Min'? Royal College (a. ) Physicians}

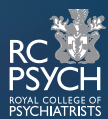

\section{during} the COVID-19 pandemic

Peer support can help staff cope with the stress that comes from their work. New guidance from the Royal College of Physicians and Royal College of Psychiatrists sets out the values, principles and practice of peer support.

Download the guidance and accompanying infographic: www.rcplondon.ac.uk/projects/peer-support-guidance

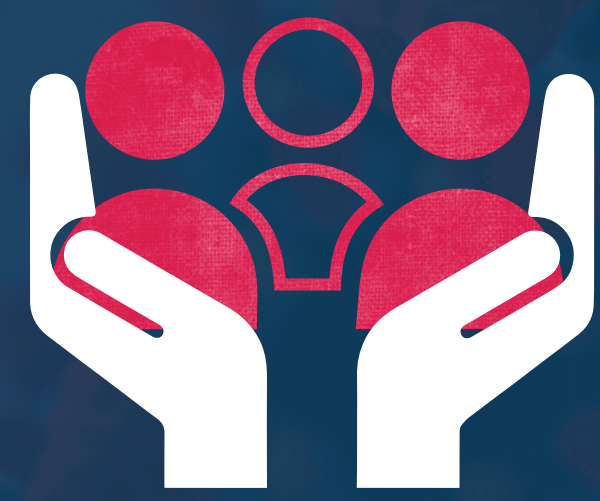

